\title{
Use of an Image-Guided Robotic Radiosurgery System for the Treatment of Canine Nonlymphomatous Nasal Tumors
}

\author{
Seth A. Glasser, DVM, DACVIM (Oncology)*, Sarah Charney, DVM, DACVIM (Oncology), DACVR (Radiation Oncology), \\ Nikolaos G. Dervisis, DVM, DACVIM (Oncology) ${ }^{\dagger}$, Matthew R. Witten, PhD, DABR (Medical Physics), \\ Susan Ettinger, DVM, DACVIM (Oncology), Jason Berg, DVM, DACVIM (Internal Medicine and Neurology), \\ Richard Joseph, DVM, DACVIM (Neurology)
}

\section{ABSTRACT}

An image-guided robotic stereotactic radiosurgery (SRS) system can be used to deliver curative-intent radiation in either single fraction or hypofractionated doses. Medical records for 19 dogs with nonlymphomatous nasal tumors treated with hypofractionated image-guided robotic stereotactic body radiotherapy (SBRT), either with or without adjunctive treatment, were retrospectively analyzed for survival and prognostic factors. Median survival time (MST) was evaluated using KaplanMeier survival curves. Age, breed, tumor type, stage, tumor size, prescribed radiation dose, and heterogeneity index were analyzed for prognostic significance. Dogs were treated with three consecutive-day, 8-12 gray (Gy) fractions of imageguided robotic SBRT. Overall MST was 399 days. No significant prognostic factors were identified. Acute side effects were rare and mild. Late side effects included one dog with an oronasal fistula and six dogs with seizures. In three of six dogs, seizures were a presenting complaint prior to SBRT. The cause of seizures in the remaining three dogs could not be definitively determined due to lack of follow-up computed tomography (CT) imaging. The seizures could have been related to either progression of disease or late radiation effect. Results indicate that image-guided robotic SBRT, either with or without adjunctive therapy, for canine nonlymphomatous nasal tumors provides comparable survival times (STs) to daily fractionated megavoltage radiation with fewer required fractions and fewer acute side effects. ( $\mathrm{J}$ Am Anim Hosp Assoc 2014; 50:96-104. DOI 10.5326/JAAHA-MS-6024)

\section{Introduction}

Nonlymphomatous nasal tumors are relatively rare in the dog, representing $1-2 \%$ of all canine neoplasms. Those nasal tumors are a heterogeneous group of tumors comprised of various histologic classifications, including mesenchymal tumors and epithelial tumors. ${ }^{1}$ Nonlymphomatous tumors tend to act in a locally aggressive manner displaying metastasis late in the course of

From the Animal Specialty Center, Yonkers, NY.

Correspondence: sglasser@animalerc.com (S.G.) disease. $^{2}$ Because of their location and biologic properties, nasal tumors are difficult to treat, and progressive local disease commonly causes deterioration in quality of life leading to either euthanasia or death. ${ }^{3,4}$

The most common treatment of nasal tumors is daily fractionated megavoltage radiation therapy, which tends to result in better survival times (STs) than either surgery or chemotherapy intensity-modulated radiation therapy; MST median survival time; SBRT stereotactic body radiotherapy; SRS stereotactic radiosurgery; ST survival time

${ }^{*}$ S. Glasser's present affiliation is Animal Emergency and Referral Associates, Fairfield, NJ.

'N. Dervisis' present affiliation is Virginia-Maryland Regional College of Veterinary Medicine, Blacksburg, VA. 
alone. $^{5-7}$ STs reported for dogs treated with megavoltage radiotherapy ( \pm surgery and/or chemotherapy) with either daily fractionated or accelerated fractionation schemes range from 8.5 mo to 19.7 mo. ${ }^{3,5,8-13}$ Moderate to severe acute and late side effects are commonly reported..$^{5,8,14,15}$ Those side effects often have a tremendously negative impact on the patient. In some cases, those side effects lead to euthanasia due to a perceived deterioration in the quality of life. An accelerated technique with nasal exenteration reported excellent long-term tumor control (median survival time [MST], $47.7 \mathrm{mo}$ ), but $38 \%$ of patients suffered osteomyelitis either with or without bone necrosis. ${ }^{11}$

Recently, daily fractionated intensity-modulated radiation therapy (IMRT) has been reported for sinonasal tumors. That treatment resulted in fewer acute and late radiation side effects, but similar STs to previous reports of daily fractionated megavoltage radiation. ${ }^{16,17}$

High total doses of radiation are required to kill tumors; however, high doses of radiation can be damaging to both tumors and normal tissues (especially late-responding normal tissues). Late-responding tissues have a greater ability to repair themselves than tumor tissues when exposed to doses (i.e., $<5$ gray [Gy]). At doses $<5 \mathrm{~Gy}$, radiation causes more damage to tumor tissues than to late-responding normal tissues. At doses $>5 \mathrm{~Gy}$, radiation causes more damage to late-responding normal tissues than to tumors. That said, doses much higher than 5 Gy are required to kill tumors. ${ }^{18}$ There are two ways to deliver the high total doses of radiation required to kill tumors while sparing late-responding normal tissues: either fractionation of the total dose or use of highly conformal techniques such as radiosurgery. Fractionation, dividing the total dose of radiation into smaller daily doses administered over several wk to $1 \mathrm{mo}$, is the most common method. Fractionated protocols in which each fraction is $<5$ Gy exploit the differential repair ability of late-responding normal cells when exposed to low dose/fraction radiation. ${ }^{18}$

All of the previously reported curative-intent radiation protocols for canine nonlymphomatous nasal tumors rely on daily fractionation of the total dose. ${ }^{3,5-12,14-17}$ That requires the use of anesthesia for each fraction and despite advances in radiotherapy techniques such as three-dimensional imaging and computerized planning, a substantial volume of normal tissue still receives a considerable dose of radiation. Three-dimensional conformal therapy and IMRT reduce the volume of normal tissue receiving a high dose of radiation, thereby limiting the damage to normal tissues but both still require daily fractionated therapy.

Stereotactic radiosurgery (SRS) and stereotactic body radiotherapy (SBRT) were developed to deliver a highly concentrated dose of radiation exclusively to the tumor. ${ }^{19}$ SRS and SBRT do not rely on the differential ability between cancer cells and lateresponding normal cells to repair damage, but rather rely on extreme conformality of the radiation to the tumor. SRS and SBRT maximize radiation to the tumor and minimize radiation exposure to the surrounding normal tissue by delivering a highly collimated beam through multiple paths intersecting the tumor volume thereby creating steep dose gradients outside the tumor. ${ }^{19}$ SRS and SBRT are delivered as either curative-intent single fractions of radiation or hypofractionated radiation (usually 3-5 fractions delivered within the same wk). That avoids lengthy fractionation protocols associated with daily fractionated radiation allowing for greater ease in patient treatment and owner compliance. SRS and SBRT have been reported for a variety of tumors in human medicine, and the use of SRS and SBRT using a University of Florida proprietary system has been reported for dogs with brain tumors and appendicular osteosarcomas. ${ }^{20,21}$

The proprietary system ${ }^{\mathrm{a}}$ is an image-guided radiosurgery system comprised of a 6 megavolt linear accelerator mounted on a robotic-controlled arm and a treatment room image-guidance system consisting of two ceiling-mounted diagnostic X-ray sources with table-mounted flat panel detectors, which provide two real-time orthogonal set-up views of the patient (Figure 1). Prior to imaging with CT, percutaneously placed fiducial markers are inserted either in or adjacent to the tumor. ${ }^{22}$ Digitally reconstructed radiographs from the planning CT are compared with data from the two real-time orthogonal set-up images. The location of the fiducial markers on the digitally reconstructed radiographs is compared with that of the fiducial markers on the two real-time orthogonal set-up images, and proprietary target localization computer software uses those comparison views to accurately position the patient for treatment via automated movements of the treatment couch. ${ }^{23}$

Treatment planning was achieved with a proprietary system ${ }^{\mathrm{b}}$ that allowed inverse planning and nonisocentric radiation delivery. The total radiation dose to be delivered to the tumor was determined, and boundaries to protect nearby critical structures were defined. That software then determined the targeting positions and the dose to be delivered from each targeting position. Once the treatment plan was optimized and approved for delivery, the target localization software previously described directed the highly mobile radiation source, which delivered highly collimated beams through multiple paths based on the treatment plan. ${ }^{22}$ In typical image-guided robotic SBRT treatment plans, approximately 100-200 beams were delivered via three paths through collimators that ranged in size from $5 \mathrm{~mm}$ to $60 \mathrm{~mm}$.

The proprietary radiosurgery system (hardware and software) delivers radiation with submillimeter accuracy. ${ }^{24-26}$ Image guided 


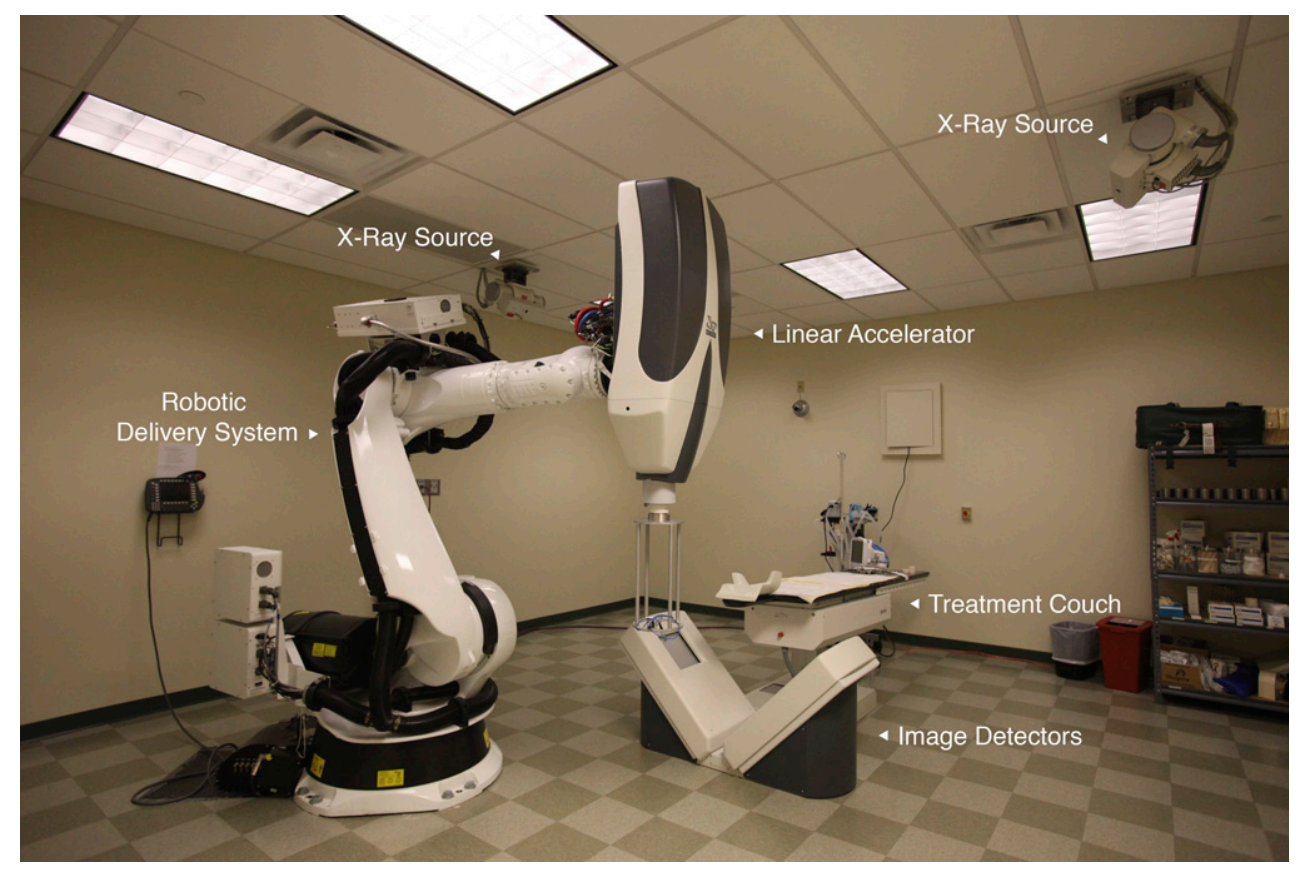

FIGURE 1 Photograph of the image-guided robotic stereotactic radiosurgery (SRS) treatment room showing the components of the image-guided robotic SRS system.

robotic SBRT has been used extensively for a wide variety of tumors in human medicine, but to the authors' knowledge, this is the first report of the use of image-guided robotic SBRT in dogs.

The purposes of this retrospective study were to evaluate the clinical outcome and prognostic factors influencing survival of dogs with spontaneously occurring nonlymphomatous nasal tumors treated with image-guided robotic SBRT either with or without adjunctive therapy and to report any associated toxicity.

\section{Materials and Methods}

Medical records were searched for dogs with spontaneously occurring nasal tumors treated with image-guided robotic SBRT at the Animal Specialty Center, Yonkers, New York, between March 2008 and August 2009. Dates were limited to that time frame to ensure adequate follow-up data were obtained. Dogs with nonlymphomatous nasal tumors treated with hypofractionated radiosurgery were eligible for inclusion in this study. Dogs diagnosed with round cell neoplasia and/or dogs treated with single-fraction radiosurgery were excluded. Follow-up information, including side effects and ST was obtained via recheck examinations at the Animal Specialty Center and/or telephone communications with clients and/or referring veterinarians.

Dogs eligible for inclusion were referred to the radiation oncology service for image-guided robotic SBRT. Varying degrees of staging prior to treatment planning were performed depending on the preferences of the referring clinicians. All animals had blood work, thoracic radiographs, and aspirates of any abnormal lymph nodes. Some animals received an abdominal ultrasound prior to therapy at the discretion of the referring clinician. The diagnosis of neoplasia was made by histopathologic confirmation. For data analysis, histologic classification was divided into sarcomas and carcinomas.

All patients underwent a computed tomography (CT) scan ${ }^{c}$ to obtain $1 \mathrm{~mm}$ slices for planning purposes. Some animals also underwent MRI for diagnostic and planning purposes. MRI was performed in cases where either clinical signs or CT imaging indicated that significant invasion into the brain was likely and if the radiation oncologist (S.C.) felt it would be useful for planning purposes. Prior to the planning CT, all patients received three or more gold fiducial markers ${ }^{\mathrm{d}}$ that were placed in the deep mucosa along the palate. Sterile placement needles ${ }^{\mathrm{e}}$ were used to place the soft tissue gold markers. All fiducial markers were placed by the radiation oncologist. The fiducial markers did not require any special expertise because the level of difficulty was similar to that of a core needle biopsy. The fiducial markers served as radiographic landmarks.

Inverse treatment planning with proprietary software ${ }^{\mathrm{b}}$ was used to create an optimized SBRT plan. In all cases, the tumor (including adjacent fluid-filled sinus cavities or nasal passages), left and right eyes and lenses, optic chiasm, pituitary gland, and brain stem were contoured. The olfactory and frontal lobes were not contoured as critical structures because there is not a known dose tolerance for those specific structures. The steep dose gradient associated with SBRT plans was relied on to minimize dose to those structures. Based on one author's experience (M.W.), using the steep gradients of SBRT to minimize dose is often used 
in human SBRT when a particular structure does not have a specified dose tolerance limit. Tumor contouring included nasal cavity disease as well as all other areas clinically determined as tumor extension based on the CT (and, in some cases, MRI) scan(s), including sinus cavities, intracranial disease, soft tissues of the head and oropharynx (gross tumor volume and clinical target volume, respectively). Tumor volume was determined using a commercial software package ${ }^{\mathrm{b}}$ based on the contoured tumor and was reported in $\mathrm{mm}^{3}$. Regional lymph nodes were not included in the treatment plan. Only one dog had regional lymph node involvement, and the node was surgically resected immediately prior to SBRT. SBRT of the node was offered, but surgery was elected by the owner. Plans were optimized by an American Board of Radiology certified medical physicist (M.W.) to deliver $95 \%$ of the prescribed dose to the entire target volume while minimizing the dose to contoured critical structures.

Total prescribed dose was based initially on maintaining equivalence to the average biologically effective dose for lateresponding tissues $\left(\mathrm{BED}_{3}\right)$ in typical daily fractionated radiation therapy protocols. Fraction size is the overriding factor in determining late effects. Specifically, the larger the fraction size, the greater the effect on late-responding tissues. ${ }^{18}$ Tolerance levels for late-responding tissues are usually set at TD5/5 meaning that the normal tissue complication probability is $<5 \% 5 \mathrm{yr}$ after radiation. ${ }^{27}$ As radiosurgery uses very high doses/fraction, late-responding tissues are at higher risk than with daily fractionated megavoltage radiation protocols. As such, starting with a conservative radiosurgery dose with a $\mathrm{BED}_{3}$ equivalent to that of the $\mathrm{BED}_{3}$ for daily fractionated megavoltage radiation seemed reasonable to ensure that late side effects to those tissues would not exceed a tolerable level. Based on one author's experience (M.W.), that method of calculation is used in the human field when starting doses for either SRS or SBRT are completely unknown.

Based on previous studies, as well as the authors' experience, it was decided that a hypothetical daily fractionated megavoltage radiation protocol of 54 Gy given in 3 Gy fractions (hypothetical protocol) was a reasonable starting point to determine a radiosurgery dose. ${ }^{13,14,16}$ Based on human radiosurgery protocols for tumors in mucosal areas, the authors elected to fractionate the total radiosurgery dose into three fractions. To determine the SBRT radiosurgery dose/fraction that would maintain an equivalent $\mathrm{BED}_{3}$ to that of the hypothetical protocol, $\mathrm{BED}_{3}$ was calculated using the following formula:

$$
\mathrm{E} / \alpha=(\mathrm{nd})[1+\mathrm{d} /(\alpha / \beta)]
$$

In that equation, $\mathrm{E} / \alpha=\mathrm{BED}$, nd $=$ total dose, and $\mathrm{d}=$ dose/ fraction. The published standard value for $\alpha / \beta$ for late-responding tissues is 3, and that value was used in all calculations reported herein. ${ }^{18}$ Using that protocol, the $\mathrm{BED}_{3}$ for the hypothetical protocol was 108 Gy. The dose/fraction for SBRT was then calculated by iteratively changing $d$, given that $n=3$ until a BED of 108 was produced. Based on that iterative calculation, a SBRT protocol of three consecutive daily fractions of $9 \mathrm{~Gy} /$ fraction for a total of 27 Gy would have an equivalent $\mathrm{BED}_{3}$ to that of the hypothetical protocol.

The time-corrected BED for acute-responding tissues and tumors $\left(\mathrm{BED}_{10}\right)$ for the hypothetical protocol was 59.6 based on the formula:

$$
\mathrm{E} / \alpha=(\mathrm{nd})[1+\mathrm{d} /(\alpha / \beta)]-0.693 / \alpha\left(\mathrm{t} / \mathrm{T}_{\mathrm{pot}}\right)
$$

In that equation, $\mathrm{E} / \alpha=\mathrm{BED}, \mathrm{nd}=$ total dose, $\mathrm{d}=$ dose $/$ fraction, $\mathrm{t}=$ total time of the protocol, $\mathrm{T}_{\mathrm{pot}}=$ the potential doubling time of the tumor, and $0.693 / \alpha\left(\mathrm{t} / \mathrm{T}_{\text {pot }}\right)=$ the correction for proliferation. The published standard value for $\alpha / \beta$ for acuteresponding tissues (including tumors) is $10, \alpha$ for the proliferation correction $=0.3$, and $\mathrm{T}_{\text {pot }}=5$ days. Those values were used in all calculations reported herein. ${ }^{18}$ A SBRT protocol of three consecutive daily fractions of $9 \mathrm{~Gy} /$ fraction for a total of $27 \mathrm{~Gy}$ would have a nontime corrected (because the dose was delivered in $<5$ days) $\mathrm{BED}_{10}$ of 51.3 . The lower $\mathrm{BED}_{10}$ of the SBRT protocol would be expected to result in fewer acute side effects and possibly less tumor control. However, the time-corrected $\mathrm{BED}_{10}$ of the above-described SBRT protocol for nasal tumors was higher than that published by Roberts et al. (1987)and LaDue et al. (1999) and similar to that published by Hunley et al. (2010). ${ }^{13,14,16}$ The physicist (M.W.) and the radiation oncologist (S.C.) involved in planning felt that the proposed protocol of three consecutive day fractions of $9 \mathrm{~Gy} /$ fraction for a total of $27 \mathrm{~Gy}$ was a reasonable starting dose, especially because it falls within the dose ranges for hypofractionated radiosurgery for head and neck tumors reported in human medicine. ${ }^{28}$

The total prescribed dose ranged between $24 \mathrm{~Gy}$ and $36 \mathrm{~Gy}$, delivered in three consecutive fractions. The variation in prescribed dose was primarily due to tumor type, with squamous cell carcinomas and anaplastic carcinomas receiving higher doses and either very large tumors or tumors with significant invasion into the brain receiving smaller doses.

Accurate patient set-up was achieved using the image-guided robotic SBRT system imaging and positioning software and hardware as described above. No immobilizers for repositioning (e.g., vacuum-lock bags, jaw immobilizers) were necessary due to the previously placed fiducial markers. Real-time imaging throughout treatment was used to ensure spatial accuracy. The radiation itself was delivered by the robotic arm-mounted linear 
accelerator as described above. The three fractions of radiation were delivered on consecutive days.

The staging scheme reported by Adams et al. (2009) was modified and used to stratify patients. ${ }^{9}$ Tumors were staged based on CT findings as follows:

$\mathrm{TI}=$ confined to one nasal passage, paranasal sinus, or frontal sinus, with no bony involvement

$\mathrm{TII}=$ any bony involvement (including bilateral nasal passage involvement) but with no evidence of orbit, subcutaneous, or submucosal mass

TIII = involvement of orbit or involvement of a subcutaneous or submucosal mass

$\mathrm{TIV}=$ tumor extension into either the nasopharynx or cribriform plate

TIVa $=$ tumor invading into brain tissue

Acute and late radiation side effects observed during follow-up examination at Animal Specialty Center or relayed by referring veterinarians or owners were recorded and scored using the Veterinary Radiation Therapy Oncology Group scheme. $^{29}$

\section{Statistical Analysis}

Overall survival analysis was performed by Kaplan-Meier productlimit method. Survival was defined as the interval between the date of treatment with SBRT and the date of death. Dogs alive at last the time of last follow-up, lost to follow-up, or whose death occurred as the result of a documented unrelated disease were censored. Patterns of survival were deemed to differ when the logrank $P$ was $\leq 0.05$. Disease-free interval was not assessed because tumor relapse was not evaluable as posttreatment CT imaging was not routinely performed. The effect on survival of independent variables in treated animals was also analyzed by the Kaplan-Meier product-limit survival analysis and Cox proportional hazards regression model. Independent variables examined were species, breed (pure or mixed), age, gender, histopathologic classification (carcinoma or sarcoma), stage, tumor volume, prescribed dose, minimum and maximum dose within the tumor, heterogeneity index (i.e., the ratio between the maximum dose within the tumor and the prescribed dose), and additional treatment (chemotherapy or radiosurgery) at the time of relapse. For continuous variables, the median was determined, and the values were subsequently grouped into two categories: above the median and below the median. Independent variables were entered in the regression model if the $P$ value was $<0.1$. All reported $P$ values were two sided. Values of $P \leq 0.05$ were considered significant. Statistical analyses were performed using a commercial software system ${ }^{\mathrm{f}}$.

\section{Results}

Nineteen dogs met the criteria for inclusion in the study. There were 13 pure-breed dogs and 6 mixed-breed dogs. The median age of patients was $9 \mathrm{yr}$ (mean, $8.8 \mathrm{yr}$; range, 4-14.1 yr). There were 11 castrated males, 4 spayed females, and 4 males. Diagnoses included adenocarcinoma $(n=9)$, carcinoma (not otherwise specified, $n=5)$, osteosarcoma $(n=1)$, chrondrosarcoma $(n=2)$, squamous cell carcinoma $(n=1)$, and anaplastic sarcoma $(n=1)$. All diagnoses were confirmed by histopathology.

Staging was based on CT scanning in all animals. The number of patients in each stage (TI, TII, TIII, TIV, TIVa) was 3, 2, 6, 3, and 5 , respectively. One patient tested positive for local lymph node metastasis at the time of treatment. That lymph node was excised prior to the first radiosurgery treatment, and metastasis was confirmed on histopathology. In total, 5 of 19 patients had clinically visible facial deformity and 3 of 19 patients had a clinically visible oral mass effect. Based on CT, 3 of 19 patients had hard palate destruction.

The median tumor volume was $7,076 \mathrm{~mm}^{3}$ (mean 7,038 $\mathrm{mm}^{3}$; range $1,11-14,384 \mathrm{~mm}^{3}$ ), the median total prescribed dose was 27 Gy (mean, 27 Gy; range, 24-36 Gy), the median minimum dose within the tumor was $20 \mathrm{~Gy}$ (mean, $21 \mathrm{~Gy}$; range, 16-31 Gy), the median maximum dose within the tumor was 32 Gy (mean, 33 Gy; range, 28-44Gy), and the median heterogeneity index was 1.27 (mean, 1.27; range, 1.22-1.35).

Thirteen patients received no additional radiation or chemotherapy at the time of relapse of clinical signs and six received either chemotherapy alone $(n=4)$ or additional SBRT with chemotherapy $(n=2)$. The two patients who received additional SBRT had CT-documented progression of their nasal tumors and were retreated with SBRT at 280 days and 448 days with three fractions of 8 Gy on three consecutive days. The STs for those two patients were 399 days and 778 days. Both of those patients presented for the initial SBRT treatment with seizures. Neither of those patients had documentable late-term side effects after either the first or second course of treatment. Both patients had documented progression of tumor prior to death.

Kaplan-Meier overall MST was 399 days (Figure 2). All dogs were included in the Kaplan-Meier analysis. Four patients were censored in the Kaplan-Meier analysis. Three animals were censored due to death of documented nontumor-related causes at 82 days (appendicular osteosarcoma), 347 days (complications associated with laryngeal paralysis), and 886 days (transitional cell carcinoma of the urinary bladder). Those dogs had stage IVa carcinoma, stage III carcinoma, and stage I carcinoma, respectively. One animal (with stage IVa carcinoma) was alive at 1,279 days. Range of ST in patients that died of disease 


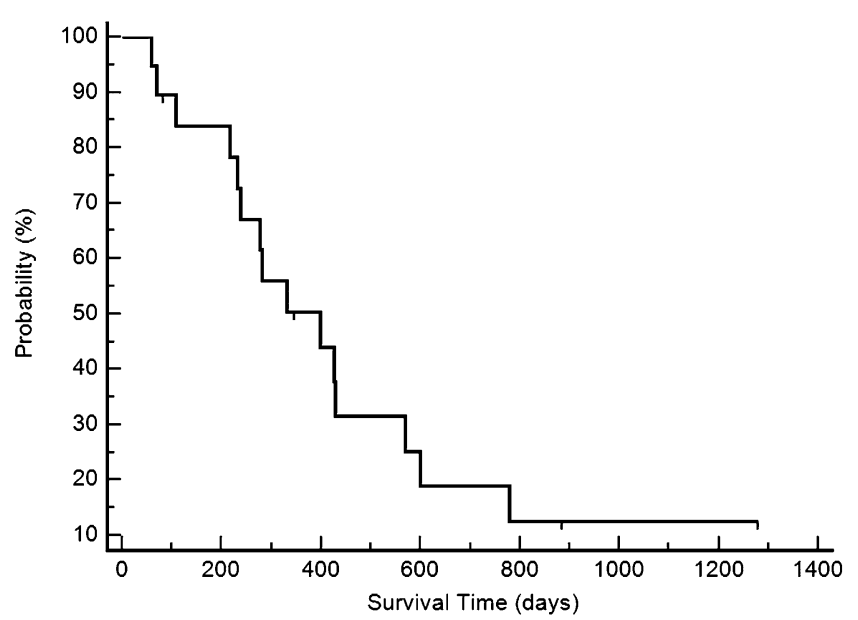

FIGURE 2 Kaplan-Meier overall survival curve for 19 patients treated with image-guided robotic stereotactic body radiotherapy (SBRT). Tick marks indicate censored animals. The median survival time (MST) was 399 days.

related to nasal tumor $(\mathrm{n}=15)$ was $59-886$ days. Those dogs had a range of stages from I to IVa, and there was a mixture of carcinomas and sarcomas. As below, stage and histopathologic classification were not prognostic. Of the 15 patients who were dead due to disease at the time of analysis, 14 died due to progressive local disease, and one patient (with stage IV carcinoma) died of metastatic disease to the lungs. One of the animals (with stage III carcinoma) with progressive disease also had severe late-term radiation side effects (oronasal fistula and bone necrosis), and the reason for euthanasia was a lack of tumor control and the degree of radiation side effects. That dog received a total prescribed dose of 30 Gy divided into three fractions.

Subjective clinical response at some time point within the first 3 mo postSBRT treatment was evaluable in all dogs. In total, 18 of 19 dogs had subjectively improved clinical response (i.e., decreased congestion, no epistaxis, decreased nasal discharge) based on owner evaluation. One dog had continued epistaxis. Five dogs had externally visible facial deformity at the time of treatment. Four of the five dogs had complete resolution of the facial deformity. One dog had continued deformity. That was also the dog that had continued epistaxis. Disease-free interval was not assessed because objective tumor relapse was not evaluable as posttreatment CT imaging was not routinely performed. CT or MRI was available in six dogs. In those dogs, tumor was either still present or had regrown at $3 \mathrm{mo}, 5 \mathrm{mo}, 13 \mathrm{mo}$, and $14 \mathrm{mo}$. In two dogs, no tumor was evident on CT or MRI at time of death/censor (Figure 3). In dogs with no postSBRT imaging, two dogs had no recurrence of clinical signs at time of death/censor, four dogs had recurrence of

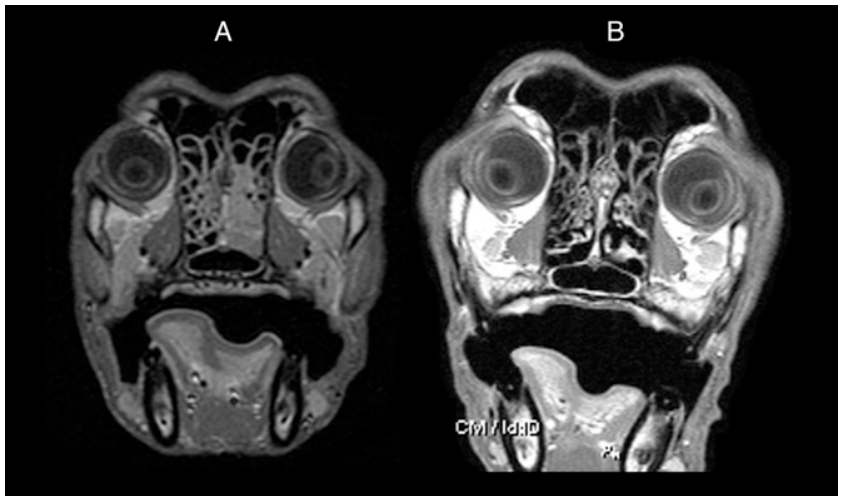

FIGURE 3 A: PreSBRT T1-weighted MRI of a patient diagnosed with left-sided nasal adenocarcinoma. B: T1-weighted MRI of the same patient obtained 678 days after image-guided robotic stereotactic treatment. Marked tumor resolution within the left nasal passage is noted. Images are displayed at the same level within the nasal cavity.

clinical signs at $2 \mathrm{mo}, 7 \mathrm{mo}, 11 \mathrm{mo}$, and $13 \mathrm{mo}$. In seven dogs, the time to recurrence of clinical signs was not available.

Of the variables analyzed for association to survival, none showed statistical significance. The overall Kaplan-Meier MST for dogs was 399 days. The MST for all carcinomas was 332 days and 485 days for all sarcomas. The MST was 778 days for stage TI patients, 418 days for TII patients, 355 days for TIII patients, 277 days for TIV patients, and 399 days for TIVa patients.

Thirteen patients $(68 \%)$ experienced no acute radiation side effects, and six patients (32\%) had very mild acute side effects. Two patients $(11 \%)$ experienced grade 1 mucous membrane toxicity and one patient (5\%) had grade 2 . There were three patients $(16 \%)$ with grade 1 skin toxicities, and one patient (5\%) with grade 1 ocular toxicity. No animals experienced grade 3 or higher acute toxicity. All acute toxicities were noted within the first 3 wk after completion of SBRT.

Late side effects were seen. The vast majority were mild and not associated with a decrease in quality of life. In total, 4 of the 19 patients $(21 \%)$ had grade 1 skin toxicity (varying degrees of alopecia, leukotrichia, and/or hyperpigmentation). One patient developed an oronasal fistula 3 mo postradiation and grade 3 bone toxicity that occurred 7 mo postradiation. In that case, there was a significant oronasal mass effect prior to treatment and it was in that location that the oronasal fistula occurred. A buccal mucosal flap was attempted 3 mo postSBRT. The flap dehisced 4 mo postSBRT and the owner elected not to pursue additional surgery. The area of the bone necrosis also had documented regrowth of tumor surrounding the area. Six patients experienced seizures in the late-term side effect period. In three of those patients, seizures 
were one of the presenting clinical complaints prior to SBRT. The remaining three patients that experienced late-term seizures had been diagnosed with stage IV or IVa disease (i.e., cribriform plate or brain invasion) at the time of treatment, and two of those three patients had a return of clinical signs associated with nasal cavity disease concurrent with the onset of the seizures. Follow-up CT scans were not performed in any of those patients, and the causes of the seizures could not be definitively determined.

\section{Discussion}

Daily fractionated megavoltage radiation therapy has been extensively reported for the treatment nonlymphomatous nasal tumors, with reported MSTs ranging from 8.5 mo to 19.7 mo. To the authors' knowledge, the use of image-guided robotic SBRT has not been reported. ${ }^{3,5,8-11}$ In the study reported herein, dogs with nonlymphomatous nasal tumors treated with image-guided robotic SBRT had an overall MST of 399 days (13.3 mo), which is within the range of reported survival for daily fractionated megavoltage radiation.

Reported prognostic factors for nasal tumors treated with daily fractionated megavoltage radiation include tumor stage, histopathologic type, facial deformity, and epistaxis., ${ }^{2,4,8,9,12,30}$ In the current study, none of the independent variables that were examined had a statistically significant effect on survival. Interestingly, in this study, stage did not have a statistically significant effect on survival even though 14 of the 19 included patients were of advanced stage (stage III, IV, or IVa), which has been shown to be a negative prognostic indicator in previous studies. ${ }^{8,9}$ Acuteand late-radiation side effects are an inherent risk with the use of any radiation modality. In studies evaluating the use of radiation for nasal tumors in animals, various radiation toxicity grading schemes have been used to characterize those side effects. ${ }^{2,8,12,14,29}$ Recently, Hunley et al. (2010) reclassified the side effects reported in several previous studies according to Veterinary Radiation Therapy Oncology Group scoring scheme for acute and lateradiation morbidity. ${ }^{8,14-16}$ The percentages of acute and late side effects for the many different daily fractionated megavoltage radiation protocols reported therein were used for comparisons with side effects in the current study.

Very few acute side effects were seen in the present imageguided robotic SBRT study, with $67 \%$ of animals experiencing no acute side effects. Eight percent of animals experienced grade 1 or grade 2 acute oral cavity toxicity. That was a substantial decrease in acute side effects compared with the 57-64\% grade 2 or grade 3 acute oral toxicity seen with daily fractionated megavoltage radiation (no dogs treated with image-guided robotic SRBT experienced grade 3 or higher acute oral toxicity). ${ }^{16}$ With image-guided robotic SBRT, $8 \%$ of animals experienced grade 1 acute cutaneous toxicity compared with $17-42 \%$ experiencing grade 2 and grade 3 cutaneous toxicity with daily fractionated megavoltage radiation. ${ }^{16}$ No dogs in the current study experienced grade 2 or higher acute cutaneous toxicity. In this study, $4 \%$ of animals experienced grade 1 acute ocular toxicity compared with $35-62 \%$ of animals experiencing grade 2 or 3 toxicity with daily fractionated megavoltage radiation. ${ }^{16}$ No dog in the current study displayed grade 2 or higher acute ocular toxicity. Acute side effects were also lower in dogs treated with IMRT than with daily fractionated megavoltage radiation, likely due to the increased conformality. Nonetheless, the IMRT acute side effects were still higher than those reported herein. ${ }^{16}$

With image-guided robotic SBRT, not only was the percentage of animals that experienced acute side effects comparatively much lower than that of daily fractionated megavoltage radiation, the severity of the acute effects was significantly less. The decrease in acute side effects may have been due to the conformality associated with image-guided robotic SBRT, but it may also be due to the lower $\mathrm{BED}_{10}$ for the image-guided robotic protocol.

Late-term radiation-induced ocular side effects (including cataracts and blindness) and skin side effects (including alopecia, leukotrichia, hyperpigmentation, and fibrosis) have been reported with daily fractionated megavoltage radiation for canine nasal tumors. In the current study, no dogs had late-term ocular effects. The low incidence of late-term grade 3 ocular toxicity compares very favorably with the $3-45 \%$ late-term grade 3 ocular toxicity seen with daily fractionated megavoltage radiation. ${ }^{16}$

Severe, potentially life threatening, late-term effects are also reported with daily fractionated megavoltage radiation (cobalt 60 ), including neurologic dysfunction (e.g., seizures) in 13\% and osteoradionecrosis in $2-5 \% .^{8,10}$ More recent reports utilizing linear accelerators with CT planning are lacking and, as such, side effects associated with those improved modalities have not been adequately explored. Accelerated protocols report osteoradionecrosis (3-14\%) and central nervous system dysfunction $(14 \%) .{ }^{11,12}$ In the current study, of the patients who experienced more severe late effect events, in most cases, it was impossible to determine whether those events were caused by tumor progression or late-term radiation effects. Six patients experienced seizures in the late-term side effect period. In three of those patients, seizures were one of the presenting clinical complaints prior to SBRT. The remaining three patients that experienced late-term seizures had been diagnosed with either stage IV or IVa disease (i.e., cribriform plate or brain invasion) at the time of treatment. Due to owner preference and associated costs, follow-up 
CT scans were not performed in any of those patients, and causes of seizures could not be definitively determined. If the seizures reported in all three of the patients for which seizures were not an initial complaint were radiation related, which is unlikely, the percentage of animals (16\%) with neurologic dysfunction postSBRT was similar to that seen with daily fractionated megavoltage radiation. One patient (5\%) developed an oronasal fistula and experienced grade 3 bone toxicity (bone necrosis). In that case, there was a significant oronasal mass effect prior to treatment and it was in that location that the oronasal fistula occurred. A buccal mucosal flap was used to fix the oronasal fistula 3 mo postSBRT. Dehiscence occurred within 1 mo of the flap closure, which appeared to be the result of expansion of the oronasal fistula. It is likely that the full extent of the tumor death and/or necrosis that caused the fistula had not occurred at the time of the surgery because it can take up to 6 mo or more for radiation-associated death of cells to occur. However, it is uncertain whether surgery for the oronasal fistula would have been successful in any case because radiation can result in a decrease in tensile strength of the mucosa, which might have prevented successful closure. The area of the bone necrosis also had documented regrowth of tumor surrounding the area, and it is difficult to determine whether the necrosis was either radiation or tumor related.

There are limitations associated with this study. The study is retrospective in nature; therefore, the authors are relying on information available in the record, the referring veterinarian's record, and/or owner recollections. The protocols used by different clinicians for staging, restaging, adjuvant therapy, and reporting of side effects varied, which could have impacted the study results, outcomes, and/or reported side effects. In addition, a relatively small number of animals were treated. As such, the fact that there were no independent variables that had a statistically significant effect on survival may be due to either the lack of power or the lack of true association. Last, posttreatment advanced imaging was not routinely performed, making it impossible to accurately assess the disease-free interval, to objectively assess the response to treatment, and to determine if some late-term side effects were tumor-related versus radiation-related.

Based on this study, it appears that any dog with nasal carcinoma or sarcoma is a potential candidate for image-guided robotic SBRT, but larger prospective studies are needed to further assess the efficacy of image-guided robotic SBRT for canine nasal tumors, to further assess prognostic factors, and to determine the optimal dose. Increasing the dose may be possible given the lack of acute side effects, but possible late effects should be further examined with serial posttreatment imaging and necropsies to determine what, if any, late-term side effects result from imageguided robotic SBRT prior to significantly increasing dose.

\section{Conclusion}

Results indicate that image-guided robotic SBRT, either with or without adjunctive therapy, for canine nonlymphomatous nasal tumors provides comparable STs as daily fractionated megavoltage radiation with fewer required fractions and fewer acute side effects. Image-guided robotic SBRT requires only three consecutive fractions of radiation in comparison with the 12-20 fractions given over several weeks that is required with daily fractionated megavoltage radiation. Severe late-term effects are possible, and the risks should be fully explained to clients.

\section{FOOTNOTES}

a CyberKnife ${ }^{\circledR}$; Accuray Inc., Sunnyvale, CA

b Multiplan ${ }^{\circledR}$ Treatment Planning System; Accuray Inc., Sunnyvale, CA

c Aquilion 16-slice C; Toshiba America Medical Systems Inc., Tustin, CA

d Gold fiducial markers; Civco Medical Solutions, Kalona, IA

e Soft tissue marker placement needles; Civco Medical Solutions, Kalona, IA

f MedCalc version 10.0.0; MedCalc Software, Ostend, Belgium

\section{REFERENCES}

1. Madewell BR, Priester WA, Gillette EL, et al. Neoplasms of the nasal passages and paranasal sinuses in domesticated animals as reported by 13 veterinary colleges. Am J Vet Res 1976;37(7):851-6.

2. Northrup NC, Etue SM, Ruslander DM, et al. Retrospective study of orthovoltage radiation therapy for nasal tumors in 42 dogs. J Vet Intern Med 2001;15(3):183-9.

3. Henry CJ, Brewer WG Jr, , Tyler JW, et al. Survival in dogs with nasal adenocarcinoma: 64 cases (1981-1995). J Vet Intern Med 1998; 12(6):436-9.

4. Evans SM, Goldschmidt M, McKee LJ, et al. Prognostic factors and survival after radiotherapy for intranasal neoplasms in dogs: 70 cases (1974-1985). J Am Vet Med Assoc 1989;194(10):1460-3.

5. Adams WM, Withrow SJ, Walshaw R, et al. Radiotherapy of malignant nasal tumors in 67 dogs. J Am Vet Med Assoc 1987;191(3): $311-5$.

6. Thrall DE, Harvey CE. Radiotherapy of malignant nasal tumors in 21 dogs. J Am Vet Med Assoc 1983;183(6):663-6.

7. Hahn KA, Knapp DW, Richardson RC, et al. Clinical response of nasal adenocarcinoma to cisplatin chemotherapy in 11 dogs. $J \mathrm{Am}$ Vet Med Assoc 1992;200(3):355-7.

8. Théon AP, Madewell BR, Harb MF, et al. Megavoltage irradiation of neoplasms of the nasal and paranasal cavities in 77 dogs. J Am Vet Med Assoc 1993;202(9):1469-75.

9. Adams WM, Kleiter MM, Thrall DE, et al. Prognostic significance of tumor histology and computed tomographic staging for radiation treatment response of canine nasal tumors. Vet Radiol Ultrasound 2009;50(3):330-5.

10. McEntee M, Page R, Heidner GL, et al. A retrospective study of 27 dogs with intranasal neoplasms treated with cobalt radiation. Vet Radiol Ultrasound 1991;32:135-9. 
11. Adams WM, Bjorling DE, McAnulty JE, et al. Outcome of accelerated radiotherapy alone or accelerated radiotherapy followed by exenteration of the nasal cavity in dogs with intranasal neoplasia: 53 cases (1990-2002). J Am Vet Med Assoc 2005;227(6):936-41.

12. Adams WM, Miller PE, Vail DM, et al. An accelerated technique for irradiation of malignant canine nasal and paranasal sinus tumors. Vet Radiol Ultrasound 1998;39(5):475-81.

13. LaDue TA, Dodge R, Page RL, et al. Factors influencing survival after radiotherapy of nasal tumors in 130 dogs. Vet Radiol Ultrasound 1999;40(3):312-7.

14. Roberts SM, Lavach JD, Severin GA, et al. Ophthalmic complications following megavoltage irradiation of the nasal and paranasal cavities in dogs. J Am Vet Med Assoc 1987;190(1):43-7.

15. Nadeau ME, Kitchell BE, Rooks RL, et al. Cobalt radiation with or without low-dose cisplatin for treatment of canine naso-sinus carcinomas. Vet Radiol Ultrasound 2004;45(4):362-7.

16. Hunley DW, Mauldin GN, Shiomitsu K, et al. Clinical outcome in dogs with nasal tumors treated with intensity-modulated radiation therapy. Can Vet J 2010;51(3):293-300.

17. Lawrence JA, Forrest LJ, Turek MM, et al. Proof of principle of ocular sparing in dogs with sinonasal tumors treated with intensitymodulated radiation therapy. Vet Radiol Ultrasound 2010;51(5): 561-70.

18. Hall EJ, Giaccia AJ. Radiobiology for the radiologist. 6th ed. Philadelphia (PA): Lippincott Williams \& Wilkins; 2006.

19. Khan FM, Gerbi BJ. Treatment planning in radiation oncology. 3rd ed. Philadelphia (PA): Wolters Kluwer/Lippincott Williams \& Wilkins Health; 2012.

20. Farese JP, Milner R, Thompson MS, et al. Stereotactic radiosurgery for treatment of osteosarcomas involving the distal portions of the limbs in dogs. J Am Vet Med Assoc 2004;225(10):1567-72, 1548.
21. Lester NV, Hopkins AL, Bova FJ, et al. Radiosurgery using a stereotactic headframe system for irradiation of brain tumors in dogs. J Am Vet Med Assoc 2001;219(11):1562-7, 1550.

22. Whyte RI, Crownover R, Murphy MJ, et al. Stereotactic radiosurgery for lung tumors: preliminary report of a phase I trial. Ann Thorac Surg 2003;75(4):1097-101.

23. Kothary N, Dieterich S, Louie JD, et al. A primer on image-guided radiation therapy for the interventional radiologist. J Vasc Interv Radiol 2009;20(7):859-62.

24. Chang SD, Main W, Martin DP, et al. An analysis of the accuracy of the CyberKnife: a robotic frameless stereotactic radiosurgical system. Neurosurgery 2003;52(1):140-6 [discussion: 146-7].

25. Antypas C, Pantelis E. Performance evaluation of a CyberKnife G4 image-guided robotic stereotactic radiosurgery system. Phys Med Biol 2008;53(17):4697-718.

26. Yu C, Main W, Taylor D, et al. An anthropomorphic phantom study of the accuracy of Cyberknife spinal radiosurgery. Neurosurgery 2004;55(5):1138-49.

27. Rubin PCG. A direction for clinical radiation pathology. In: Vaeth J, ed. Frontiers of radiation therapy and oncology VI. Baltimore (MD): University Park Press; 1972.

28. Ryu SI, Chang SD, Kim DH, et al. Image-guided hypo-fractionated stereotactic radiosurgery to spinal lesions. Neurosurgery 2001;49(4): 838-46.

29. Ladue T, Klein MK. Toxicity criteria of the veterinary radiation therapy oncology group. Vet Radiol Ultrasound 2001;42(5):475-6.

30. Rassnick KM, Goldkamp CE, Erb HN, et al. Evaluation of factors associated with survival in dogs with untreated nasal carcinomas: 139 cases (1993-2003). J Am Vet Med Assoc 2006; 229(3):401-6. 